CentroSur

Social Science Journal

\section{Evaluación socioeconómica de la producción de jengibre en la zona norte de la provincia de Los Ríos}

\section{Socioeconomic evaluation of ginger production in the northern area of the Los Ríos province}

Centro Sur.

Social Science Journal

Abril 2020 - E3

http://centrosureditorial.com/ind ex.php/revista

eISSN: 2600-5743

revistacentrosur@gmail.com

Recepción: 4 de marzo 2018

Aprobación 12 junio 2019

Pag 223 - 235

Atribución/Reconocimiento-

NoComercial-Compartirlgual 4.0

Licencia Pública Internacional -

CC BY-NC-SA 4.0

https://creativecommons.org/lice nses/by-nc-sa/4.0/legalcode.es

\begin{abstract}
Resumen
Actualmente los procesos de producción, comercialización y exportación en el mercado nacional e internacional, debido al incremento de la demanda externa de productos agrícolas, principalmente del jengibre el cual tiene una gran apreciación en el mercado internacional, debido a las múltiples utilizaciones que se le puede dar a este producto en el sector alimenticio, cosméticos, y medicina. Este producto es cultivado en las zonas subtropicales de nuestro país como: Los Ríos entre otras provincias. La producción y comercialización de jengibre generan empleo directo para los pequeños agricultores y para los habitantes de las zonas rurales por motivo de que el cultivo demanda
\end{abstract}

mucha cantidad de trabajadores. Claramente vimos que el $100 \%$ de agricultores no reciben ayuda técnica

\footnotetext{
1.Magister, Universidad Técnica Estatal de Quevedo. Quevedo, Ecuador. ORCID. https://orcid.org/0000-00016656-3381 Email. ealvarez@uteq.edu.ec. Google académico.

https://scholar.google.es/citations?hl=es\&user=w9rRn8AAAAJ

2. Estudiante, Universidad Técnica Estatal de Quevedo. Quevedo, Ecuador. ORCID https://orcid.org/0000-00028323-5117 Email. Yomira.alava2015@uteq.edu.ec. Google académico.

https://scholar.google.es/citations?hl=es\&user=kv0RcAM AAAAJ

3 Magister, Universidad Técnica Estatal de Quevedo. Quevedo, Ecuador. ORCID. https://orcid.org/0000-00031822-7839 Email. Jaime.orellana@uteq.edu.ec. Google académico.

https://scholar.google.es/citations?hl=es\&user=PkFrie8AA AAJ

4. Magister, Universidad Técnica Estatal de Quevedo. Quevedo, Ecuador. ORCID. https://orcid.org/0000-00025332-5365 Email. taniatipan@uteq.edu.ec. Google académico.

https://scholar.google.es/citations?hl=es\&user=dFRNgSg AAAAJ
} 
de ninguna institución, ya sea pública como privada; y el manejo que realizan los productores del jengibre en las labores culturales es de manera manual y química.

Palabras clave: Jengibre, internacional, producción, pequeños agricultores, demanda.

\section{ABSTRACT}

Currently the processes of production, marketing and export in the national and international market, due to the increase in external demand for agricultural products, mainly ginger which has a great appreciation in the international market, due to the multiple uses that can be Give this product in the food, cosmetics, and medicine sector. This product is cultivated in the subtropical areas of our country such as: Los Ríos among other provinces. The production and commercialization of ginger generate direct employment for small farmers and for the inhabitants of rural areas because the crop demands a lot of workers. We clearly saw that $100 \%$ of farmers do not receive technical assistance from any institution, whether public or private; and the management of ginger producers in cultural activities is manual and chemical.

Key words: Ginger, international, production, small farmers, demand.

\section{Introducción}

A nivel mundial la producción de jengibre se centra en países asiáticos. China es el mayor productor de jengibre quien cubre la demanda en mercados europeos y estadounidenses, su consumo es principal en la alimentación debido a sus altos beneficios que contiene ayudando a su salud, ya que contiene aceites naturales, extraídos de la raíz, también posee propiedades inflamatorias, expectorantes y antisépticas. (Nuñez, 2019)

En Ecuador el jengibre es cultivado en zonas subtropicales como Santo Domingo de los Tsáchilas, Esmeraldas, Morona Santiago, Napo, Guayas, Los Ríos y Pichincha. (Oscullo Cattani, 2011). Ecuador es un país de gran potencial agrícola, con un aporte del $8 \%$ del PIB, contribuyendo así a la obtención de empleo en zonas rurales a través de la contratación de la mano de obra es parte de producción agropecuaria y de esta manera cubre el desarrollo socioeconómico de los habitantes. (Nuñez, 2019)

En la provincia de Los Ríos su economía se caracteriza por la agricultura que posee, además el clima favorece la producción de los distintos productos agrícolas como el cacao, banano, maíz y arroz, gracias a su ubicación geográfica le ha permitido en convertirse en una potencia agrícola y productiva, ya que cuenta con suelos fértiles y recursos hídricos. (Nuñez, 2019) 
Este artículo tiene como objetivo analizar la evaluación socioeconómica de la producción de jengibre en la provincia de Los Ríos zona norte, las cuales pertenece a Mocache, Buena Fe, Quevedo, Valencia para dar a conocer

\section{Origen del jengibre}

El nombre de jengibre proviene del sánscrito Springavera, el nombre se aplicó debido a la forma de la raíz. Esta raíz se conoce como una de las especies con aroma de uso casero más antigua, ya que su cultivo se remonta a más de 4500 años en India y al sur de China. Fue introducido en el continente asiático a Europa en el siglo IX por los árabes, después en el siglo XIII navegantes árabes. (Moreta Quilca, 2016)

El jengibre es una planta herbácea, con porte de caña, contiene gran cantidad de células secretoras las cuales están aisladas en el seno del tejido de los órganos. Está dotada de un rizoma subterráneo irregular, llamado mano o pata y de ahí parten vástagos aéreos que son lanceoladas. (Bandile, 2015). Los rizomas del jengibre son troncos monopodiales de $50 \mathrm{~cm}$ de lago o más, son achatados, enteros o divididos como dedos de mano. (Tello, 2014)

\section{Aplicaciones del Jengibre}

El jengibre es comercializado internacionalmente como jengibre deshidratado y fresco la cual consta de diferentes presentaciones. El uso del jengibre son varias, como en la comida, que se usa como saborizante y aromatizante. También se usa el jengibre como especia tanto como para propósitos culinarios como alimentos procesados y en pastelería, confitería, preparaciones cárnicas, etc. (Marcelo López Piscocama, 2016) Para extraer oleorresina, se lo extrae del jengibre seco con solventes orgánicos. La oleorresina brinda factores organolépticos fundamentales del rizoma, la esencia del aceite y los principios pungentes, incluido ácidos grasos, carbohidratos y resinas. La aplicación de las oleorresinas es idéntica a las de la especia, pero principalmente en embutidos y en algunas bebidas. (Moreta Quilca, 2016)

Este producto posee el aroma, pero no la pungencia. Es utilizado en bebidas alcohólicas y no alcohólicas, usado también en confitería, perfumería y adicionado a la oleorresina para reiniciar un balance entre la fragancia y la pungencia desaparecida en el proceso de extracción. (Oscullo Cattani, 2011)

En lo que concierne a la medicina, existen muchos beneficios, de acuerdo a (Oscullo Cattani, 2011), es utilizado en caso de dispepsia, flatulencia y dolor de estómago. EI jengibre contiene compuestos parecidas a las enzimas digestivas que de tal manera ayudan a la digestión de comidas ricas en proteínas. También es bueno para quitar las náuseas, vértigo, mareo y vómitos. (Bandile, 2015) 
(Polo, 2018) Indica que el jengibre es un buen quemador de grasas ya que provoca la pérdida de apetito, ayuda a reducir la anemia, artritis, resfrío, tos, influenza, fiebre. También se usa para promover el flujo de la bilis, también como antioxidante y anticoagulante que ayuda a reducir el colesterol. También se ha probado que el jengibre posee actividad antimutagénica.

\section{El cultivo del Jengibre}

\section{Clima}

El jengibre crece normalmente en climas subtropicales y tropicales, su temperatura ambiente oscila entre $18^{\circ} \mathrm{C}$ a $32^{\circ} \mathrm{C}$, la más favorable entre $22^{\circ} \mathrm{C}$ y $28^{\circ} \mathrm{C}$, una humedad relativa del $80 \%$ aproximadamente, la cual le permite desarrollarse normalmente, se desarrolla también en zonas soleadas y en regiones tropicales, necesita u8na pluviosidad de 1500 a 2500mm anuales. (Dixon et al., 2015)

\section{Suelo}

Los suelos adecuados para el cultivo del jengibre son aquellos ricos en materia orgánica y son drenajes ligeros como un suelo franco-arsénico, este ayuda su libre desarrollo y elimina la posibilidad de deteriorarse o podrirse, con pH entre 5,5 y 7,5. No se recomiendan sembrar en suelos arenosos o muy gruesos y arcillosos, ya que esto constituye a que la plantación no evolucione como se espera y la consecuencia de ello se obtendrán rizomas en cantidad y peso limitados, por lo cual el producto no sería rentable para los productores. (Marcelo López Piscocama, 2016)

\section{Propagación}

La reproducción del jengibre se realiza por vía asexual, esto se realiza dividiendo los tallos portadores de al menos una yema y mide de 3 a 5 centímetros de longitud. (Morales, 2017). La tarea de seccionar o trazar los rizomas, se debe realizar anticipado de cuatro a cinco días antes de sembrarlo, esto consiste que la superficie cortada se seque y no entre en estado de descomposición. (Morales, 2017)

\section{Comercialización}

La progresiva integración de los mercados internacionales y su analogía con la política han realizado nuevas combinaciones de comercio, inversión y convenios para expandirse a nuevos mercados. Acotando a todo ello, existen impedimentos para entrar a mercados como instrumentos de política comercial, las remuneraciones a la inversión foránea y a la falta de políticas de competitividad, estos son algunos de las trabas para generar ganancias que brindan las economías de escala. (Marcelo López Piscocama, 2016) 
Por lo que es fundamental e inmediato crear políticas de competencia en el sector agrícola que garanticen mejores condiciones e incentiven el uso fundamental de nuevas tecnologías como es la agricultura dinámica, que ayudará a la creación de diversas fuentes de empleo y desarrollo económico. Además de evitar posibles prácticas anticompetitivas del sector frente a otros sectores de la economía. (Moreta Quilca, 2016)

Los supermercados son una fuente fundamental para comercializar el producto, esto se debe que el $47 \%$ de los consumidores lo adquieren en estos lugares. También se puede realizar la venta directa del jengibre a los consumidores a través de ferias libres y ofrecer un servicio a domicilio, el cual se entregue el producto, estas son propuestas para la comercialización del mismo. (Oscullo Cattani, 2011)

\begin{tabular}{|c|c|c|c|c|c|c|}
\hline \multicolumn{2}{|c|}{ EXPORTACIONES JENGIBRE (KION) } & & \multirow[t]{2}{*}{6} & \multirow{2}{*}{\multicolumn{3}{|c|}{2,014}} \\
\hline \multirow[b]{2}{*}{ MES } & \multicolumn{2}{|r|}{2,015} & & & & \\
\hline & FOB & KILOS & $\begin{array}{l}\text { PREC. } \\
\text { PROM }\end{array}$ & FOB & KILOS & $\begin{array}{l}\text { PREC. } \\
\text { PROM }\end{array}$ \\
\hline ENERO & $1,592,549$ & 633,455 & 2.51 & $1,090,809$ & 441,995 & 2.47 \\
\hline FEBRERO & $1,249,350$ & 456,135 & 2.74 & $1,171,980$ & 489,671 & 2.39 \\
\hline MARZO & 776,444 & 305,002 & 2.55 & 870,059 & 440,485 & 1.98 \\
\hline ABRIL & 451,680 & 185,425 & 2.44 & 436,564 & 198,367 & 2.20 \\
\hline MAYO & 403,291 & 191,313 & 2.11 & 497,452 & 274,201 & 1.81 \\
\hline JUNIO & 738,298 & 360,863 & 2.05 & $1,193,379$ & 635,662 & 1.88 \\
\hline JULIO & & & & $1,673,427$ & 879,906 & 1.90 \\
\hline AGOSTO & & & & $3,316,156$ & $1,561,363$ & 2.12 \\
\hline SEPTIEMBRE & & & & $3,723,527$ & $1,820,908$ & 2.04 \\
\hline OCTUBRE & & & & $4,180,102$ & $1,942,483$ & 2.15 \\
\hline NOVIEMBRE & & & & $4,594,325$ & $1,897,623$ & 2.42 \\
\hline DICIEMBRE & & & & $3,814,770$ & $1,537,947$ & 2.48 \\
\hline TOTALES & $5,211,612$ & $2,132,193$ & 2.44 & $26,562,550$ & $12,120,611$ & 2.19 \\
\hline PROMEDIO MES & 868,602 & 355,366 & & $2,213,546$ & $1,010,051$ & \\
\hline$\%$ CRECIMIENTO ANUAL & $-61 \%$ & $-65 \%$ & $12 \%$ & $254 \%$ & $277 \%$ & $-6 \%$ \\
\hline
\end{tabular}

Gráfico 1: Estadísticas de los países a los cuales se ha exportado el producto. Fuente: 2015. Elaborado: Los Autores 


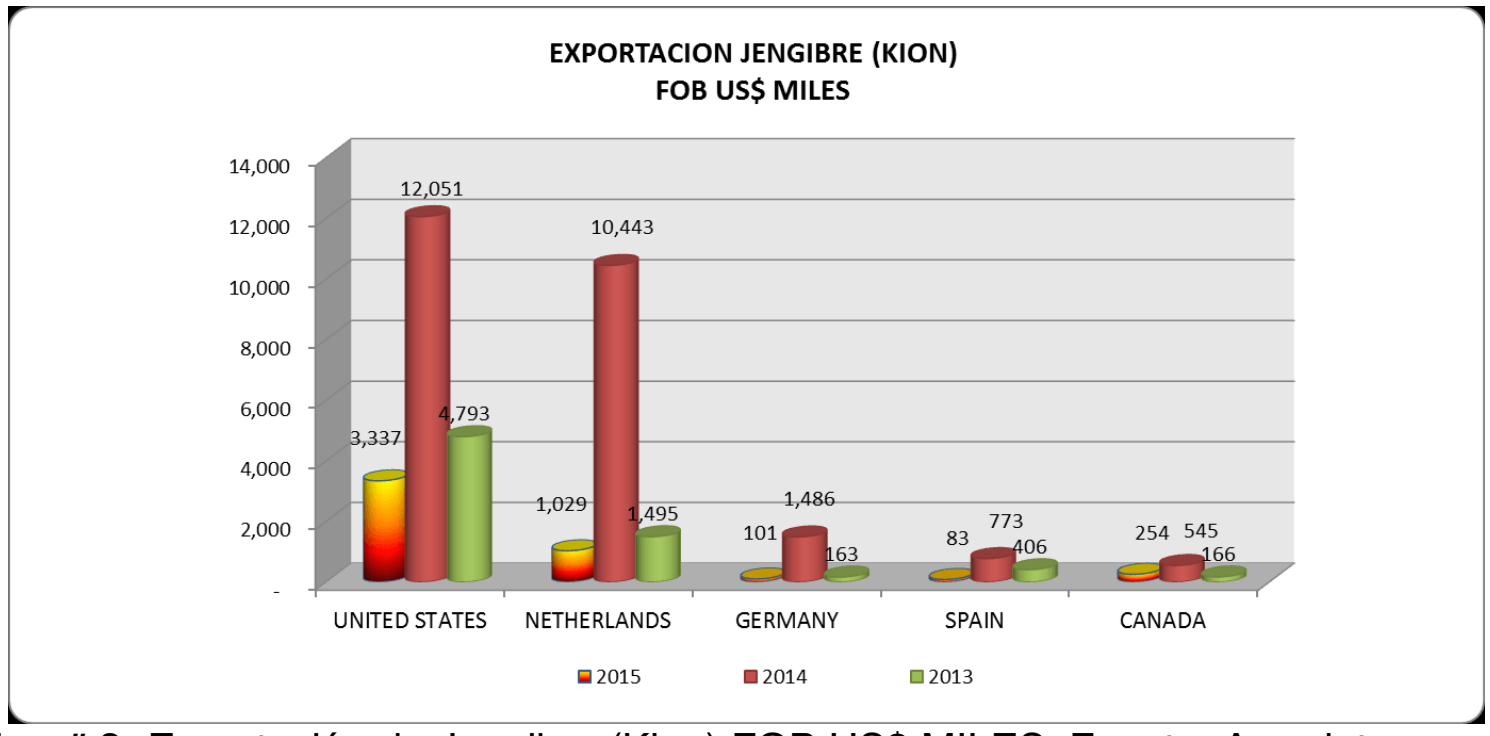

Gráfico \# 2: Exportación de Jengibre (Kion) FOB US\$ MILES. Fuente: Agrodata Elaborado: Los Autores

La exportación de jengibre en el 2015 alcanza los U\$ 5.2 millones a un precio en alza de U\$ 2.44 kilo promedio.

A Estados Unidos se vende (64\% del total), U\$ 3.3 millones, le sigue Holanda con U\$ 1.0 millones (20\%).

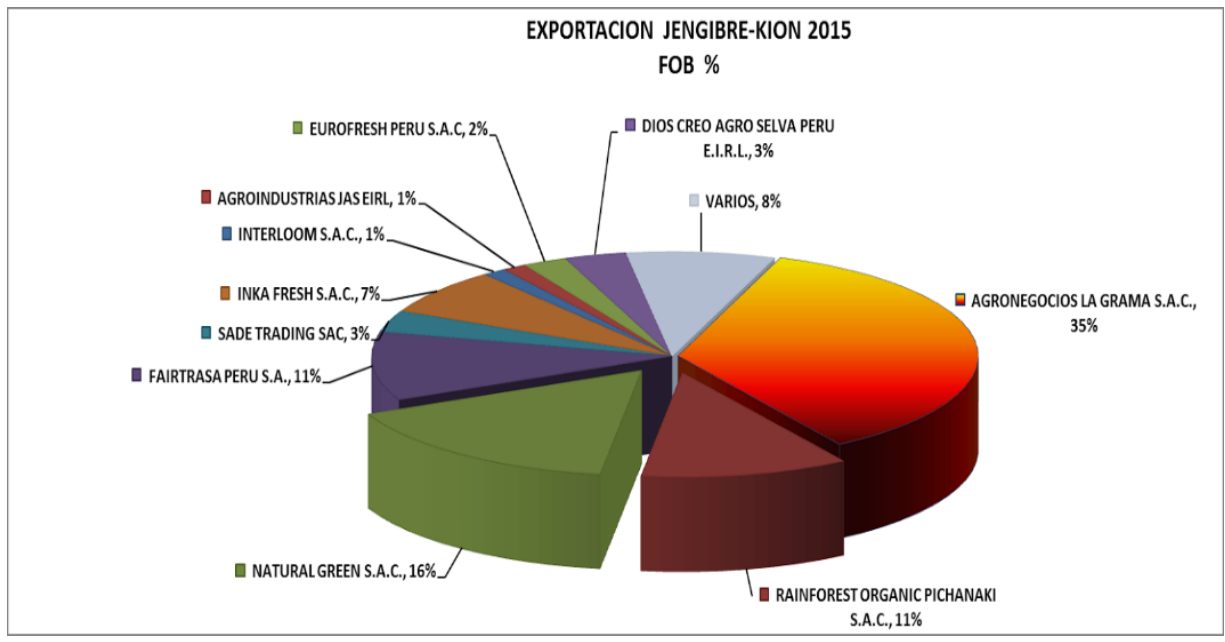

Gráfico \# 3: Ranking de las 10 empresas que más han exportado/importado el producto 
Ocho mil quinientas libras de jengibre, que provienen de dos importantes comunidades situadas en la frontera del cantón Taisha, fueron comercializadas a una entidad nacional intención de exportación, tras convenios comerciales que tramitaron técnicos del Proyecto de la Agenda de Transformación Productiva para la Amazonía, que promueve el Ministerio de Agricultura y Ganadería (MAG), en Morona Santiago. El jengibre es una raíz de origen oriental. Su siembra se da en situaciones naturales y sin mucha demanda de cuidado. Este producto es muy rentable y altamente demandado, por las propiedades que contiene dentro de la medicina cotidiana; debido a su peculiar sabor y aroma se usa para múltiples preparaciones gastronómicas. Para la traslación de este producto se facilitó dos botes del MAG, lo que simboliza un gran ahorro para las trece familias shuar que comercializaron el jengibre.

Sin embargo, el Ministerio de Agricultura y Ganadería, apoya en la nueva búsqueda de mercados y la combinación logística para trasladar los productos. Las embarcaciones comenzaron sus actividades en junio del 2015 para ayudar a los agricultores en la buena comercialización de sus productos, envío maquinaria e insumos, así como el traslado de técnicos del MAG hasta las parroquias y comunidades de la frontera de los cantones Taisha y Tiwintza. (Ministerio de Agricultura y Ganadería)

\section{Materiales y métodos}

La investigación es de tipo exploratoria, ya que ofrece un primer acercamiento al problema que se estudia y conoce. Esta investigación se realiza para conocer el tema que se abordará, la que les permita estas más en contacto con aquello que hasta el momento se desconocía. Los resultados de la investigación exploratoria, les dan una vista o comprensión superficial del tema, pero es un paso fundamental inevitable para cualquier tipo de investigación que se quiera llevar a cabo.

Con este tipo de investigación, se obtiene la indagación inicial para prolongar con una investigación más rigurosa, o se deja planteada y formulada la hipótesis. Entre las estrategias que se utilizan será la encuesta, esta consiste en reunir datos a través de la entrevista con personas, las encuestas se realizaron a las personas de la zona norte, por correo, teléfono o por internet.

Esta investigación se llevó a cabo en la provincia de Los Ríos zona norte que son Valencia, Buena fe, Mocache y Quevedo, año 2020. El objetivo del cual es indagar sobre la evaluación socioeconómica de la producción de jengibre realizando encuestas a los agricultores de las zonas mencionadas. 


\section{CALCULO TAMAÑO DE MUESTRA}

Fórmula:

$$
\boldsymbol{n}=\frac{N * Z_{\alpha}^{2} * p * q}{e^{2} *(N-1)+Z_{\alpha}^{2} * p * q}
$$

$\boldsymbol{n}=$ Tamaño de muestra buscado

$N=$ Tamaño de la Población o Universo

$\mathrm{Z}=$ Parámetro estadístico que depende el Nivel de Confianza (NC)

$e=$ Erro de estimación máximo aceptado

$p=$ Probabilidad de que ocurra el evento estudiado (éxito)

$q=(1-p)=$ Probabilidad de que no ocurra el evento estudiado

\begin{tabular}{|l|l|l|l|l|}
\hline & & & & \\
\hline & & & \\
\hline Parámetro & Valor & & Tamaño de muestra & \\
\hline N & 694.452 & & "n" = & \\
\hline Z & 1,960 & 383,95 & \\
\hline P & $50,00 \%$ & & & \\
\hline Q & $50,00 \%$ & & & \\
\hline e & $5,00 \%$ & & & \\
\hline & & & & \\
\hline
\end{tabular}

\section{Resultados}

En la tabla 1, se observar el tamaño de la población que cultiva el jengibre

Tabla 1. Nivel de Instrucción del productor

\begin{tabular}{l|l|l} 
OPCIONES & FRECUENCIA & $\%$ \\
\hline Primaria & 62 & 62 \\
\hline Secundaria & 32 & 32 \\
\hline Técnico & 6 & 6 \\
\hline Tercer nivel & 0 & 0
\end{tabular}


Total

100

100

Fuente: Encuestas aplicadas. Elaborado por: Autores

Dentro de los 100 encuestados el $62 \%$ de los productores han terminado sus estudios en la primaria, el $32 \%$ han realizado la secundaria, y sólo el $6 \%$ tienen un estudio técnico, y ninguno tiene estudios universitarios.

Tabla 2. Identificación étnica

\begin{tabular}{l|l|l} 
OPCIONES & FRECUENCIA & $\%$ \\
\hline Blanco & 14 & 14 \\
\hline Mestizo & 57 & 57 \\
\hline Montubio & 29 & 29 \\
\hline Total & 100 & 100
\end{tabular}

Fuente: Encuestas aplicadas

Elaborado por: Autores

Según los resultados de la tabla \# 2 se obtuvo el 14\% las personas encuestadas son de etnia blanca, un $57 \%$ de los encuestados son de etnia mestizo, el $29 \%$ de los encuestados son de etnia montubia.

Tabla 3. Tipo de vivienda

\begin{tabular}{l|l|l|l|l}
\multirow{2}{*}{ OPCIONES } & PROPIA & & ARRENDADA & \\
\cline { 2 - 5 } & Frecuencia & $\%$ & Frecuencia & $\%$ \\
\hline Cemento & 38 & 38 & 20 & 20 \\
\hline Madera & 9 & 9 & 3 & 3 \\
\hline Mixta & 23 & 23 & 7 & 7 \\
\hline Total & 70 & 70 & 30 & 30
\end{tabular}




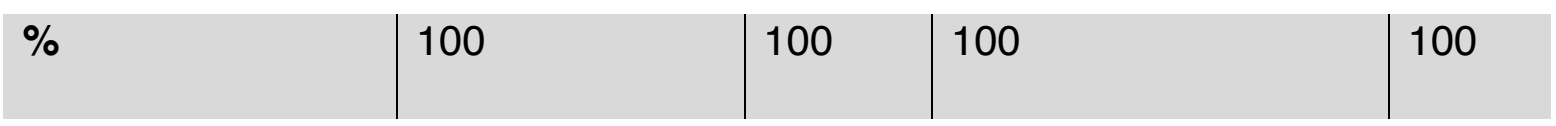

Fuente: Encuestas aplicadas. Elaborado por: Autores

El tipo de vivienda de los productores arrojan un resultado del $38 \%$ de los productores tienen casa propia de cemento y el $20 \%$ arriendan. El $9 \%$ tienen casa propia de madera y el $3 \%$ arriendan. El $23 \%$ de los productores tienen casa mixta propia y el $7 \%$ arriendan.

Tomamos en cuenta que la mayoría de los productores encuestados tienen casa propia.

Tabla \# 4. Recibe asistencia técnica por parte de alguna institución estatal o privada, lleva registro contable de la producción

\begin{tabular}{l|l|l|l|l}
\multirow{2}{*}{ Opciones } & $\begin{array}{l}\text { Asistencia } \\
\text { técnica }\end{array}$ & & Registro contable & \\
\cline { 2 - 5 } & Frecuencia & $\%$ & Frecuencia & $\%$ \\
\hline Si & 0 & 0 & 0 & 0 \\
\hline No & 100 & 100 & 100 & 100 \\
\hline Total & 25 & 100 & 25 & 100
\end{tabular}

Fuente: Encuestas aplicadas. Elaborado por: Autores

En la tabla \# 4observamos que el $100 \%$ de los productores encuestados no reciben ninguna ayuda de parte del gobierno, o institución privada, además se tiene en cuenta que nadie tiene un registro contable de la producción, lo realizan de manera empírica.

Tabla 5. Fuentes de financiamiento para el cultivo

\begin{tabular}{l|l|l} 
OPCIONES & FRECUENCIA & $\%$ \\
\hline Recursos propios & 100 & 100 \\
\hline Préstamo & 0 & 0 \\
\hline Total & 100 & 100
\end{tabular}

Fuente: Encuestas aplicadas 
Para el sembrío y cosecha del jengibre se da a notar que la mayoría de los encuestados de la zona norte de la ciudad de Los Ríos el 100\% lo realza con sus recursos propios sin solicitar préstamo alguno en alguna entidad financiera.

Tabla 6. Principales problemas que afectaron la actividad

\begin{tabular}{l|l|l} 
OPCIONES & FRECUENCIA & $\%$ \\
\hline Financiamiento & 11 & 11 \\
\hline Exceso de oferta & 23 & 23 \\
\hline Precio del producto & 66 & 66 \\
\hline Total & 100 & 100
\end{tabular}

Fuente: Encuestas aplicadas. Elaborado por: Autores

Unos de los principales problemas que afectaron la actividad de los productores de jengibre es el precio del producto con un porcentaje de $66 \%$. El $23 \%$ de los encuestados indicaban que el exceso de oferta, y el otro $11 \%$ nos indicó que era el financiamiento.

Tabla \# 7. Propiedad de la tierra y análisis del suelo

\begin{tabular}{l|l|l|l|l|l}
\multicolumn{2}{l|}{ Propiedad de la Tierra } & \multicolumn{4}{l}{ Análisis del suelo } \\
\hline Opciones & Frecuencia & $\%$ & Opciones & Frecuencia & $\%$ \\
\hline Arrienda & 68 & 68 & Realiza & 47 & 0 \\
\hline Propio & 32 & 32 & No realiza & 53 & 100 \\
\hline Total & 100 & 100 & Total & 25 & 100
\end{tabular}

En la tabla \# 7 da como resultado que el $68 \%$ de los productores arrienda terrenos y el $32 \%$ tienen terreno propio. En cuanto al análisis del suelo el $47 \%$ de los encuestados realiza análisis, mientras que el $53 \%$ no lo hace. 
Tabla \# 8. Comercialización del producto

\begin{tabular}{l|l|l|} 
Opciones & Frecuencia & $\%$ \\
\hline Acopiadores & 22 & 22 \\
\hline Intermediarios & 78 & 78 \\
\hline Exportadores & 0 & 0 \\
\hline Total & 100 & 100
\end{tabular}

En la comercialización del producto, ninguno de los productores exporta el jengibre, mientras que el $78 \%$ utiliza intermediarios para vender el producto, y el $22 \%$ acopia el jengibre para luego su venta.

\section{Conclusiones}

A través de la revisión de la literatura se logró conocer las divisas características y beneficios que contiene el jengibre, que es base primordial para esta investigación, el mismo que indica el tiempo que dura la cosecha para lograr obtener la materia prima y un producto final de buena calidad. La producción y comercialización de jengibre generan empleo directo para los pequeños agricultores y para los habitantes de las zonas rurales por motivo de que el cultivo demanda mucha cantidad de trabajadores. Claramente vimos que el $100 \%$ de agricultores no reciben ayuda técnica de ninguna institución, ya sea pública como privada; y el manejo que realizan los productores del jengibre en las labores culturales es de manera manual y química.

\section{Referencia}

Dixon, J., Gulliver, A., \& Gibbon, D. (2015). Sistemas de producción agropecuaria y pobreza. Cómo mejorar los medios de subsistencia de los pequeños agricultores en un mundo cambiante. In FAO - Food and Agriculture Organization of the United Nations.

Marcelo López Piscocama, L. C. M. (2016). Escuela Superior Politécnica ". ( Reglamento de Exámenes y Títulos Profesionales de la.

Morales, A. (2017). El Cultivo del Jengibre. Ministerio De Agricultura Y Ganaderia Republica De Costa Rica. 
http://www.mag.go.cr/bibioteca_virtual_ciencia/manual-jengibre-pz.pdf Moreta Quilca, E. (2016). "Estudio de factibilidad para la creación de una microempresa productora y comercializadora de jengibre en la comunidad de Parambas, parroquia Lita, cantón Ibarra, provincia de Imbabura."

Nuñez, A. K. L. (2019). Universidad técnica estatal de quevedo facultad de ciencias pecuarias.

Oscullo Cattani, P. Á. (2011). Estudio de factibilidad para la producción y comercialización de jengibre (Zingiber officinale Roscoe) variedad hawaiano, en San Lorenzo provincia de Esmeraldas. 136.

http://repositorio.usfq.edu.ec/bitstream/23000/1237/1/101833.pdf

Polo, B. A. R. (2018). Procesamiento de Jengibre Fresco Orgánico Para Exportación. 48.

http://repositorio.lamolina.edu.pe/bitstream/handle/UNALM/3487/refulio-polobenny-alberto. pdf?sequence $=1$ \&isAllowed=y

Tello, M. (2014). Estudio de las aplicaciones terapéuticas Del jengibre. Botanic, 61. http://dspace.ucacue.edu.ec/bitstream/reducacue/6543/1/Estudio de las apliciones terapéuticas del jengibre.pdf 